\title{
First-principles study and modeling of strain-dependent ionic migration in $\mathrm{ZrO}_{2}$
}

\author{
J. A. Hirschfeld* \\ Forschungszentrum Jülich, IAS, DE-52425 Jülich, Germany \\ H. Lustfeld \\ Forschungszentrum Jülich, PGI, DE-52425 Jülich, Germany
}

(Received 9 September 2011; revised manuscript received 9 December 2011; published 27 December 2011)

\begin{abstract}
Electrolytes with high ionic conductivity at lower temperatures are the prerequisite for the success of Solid Oxide Fuel Cells (SOFC). One promising candidate is doped zirconia. In the past its ionic conductivity has mainly been increased by decreasing its thickness. However, the influence of the thickness is only linear, whereas the impact of migration barriers is exponential. Therefore understanding the oxygen transport in doped zirconia is of fundamental importance. In this work we pursue the approach of the strain dependent ionic migration in zirconia. We investigate how the migration barriers for oxygen ions respond to a change of the atomic strain. We employ the method of Density Functional Theory (DFT) calculations to relax the atomic configurations to the ground state. In connection with the Nudged Elastic Band (NEB) method we obtain the migration barrier of the oxygen ion jumps in zirconia for a given lattice constant. Similar to other publications we observe a decrease in the migration barrier for expansive strain, but in addition we also find a migration barrier decrease for high compressive strains beyond a maximal height of the migration barrier at an intermediate compressive strain. We present a simple analytic model which, by using interactions of the Lennard-Jones type, gives an explanation for this behavior.
\end{abstract}

DOI: $10.1103 /$ PhysRevB.84.224308

PACS number(s): 82.45.Gj, 82.45.Xy, 82.20.Pm, 31.15.E-

\section{INTRODUCTION}

Among fuel cells the high temperature Solid Oxide Fuel Cell (SOFC) is a very promising candidate due to its high power density and its wide variety of possible fuel types (hydrogen, $\mathrm{CO}$, methane, ammonia, etc.). At present one problem of the SOFC is the required high temperature $\left(T>700^{\circ} \mathrm{C}\right)$. The standard electrolyte being used, the Yttria Stabilized Zirconia $\mathrm{Y}_{2 x} \mathrm{Zr}_{1-2 x} \mathrm{O}_{2-x}$ (YSZ), has a negligible ionic conductivity below $700{ }^{\circ} \mathrm{C}$ and the progress from the $800^{\circ} \mathrm{C}$ SOFC to the $700^{\circ} \mathrm{C}$ SOFC was mainly achieved by fabricating extremely thin $^{1}$ electrolyte layers. Nowadays electrolyte thicknesses of $10 \mu \mathrm{m}$ are possible. To construct a mechanically stable and gas-tight layer of that thickness is however a challenging task.

It seems that decreasing the thickness of the electrolyte further will reach its limits in a not too far future. Therefore, it is necessary to look for alternative approaches overcoming the problem of the high operating temperatures in SOFCs. A prerequisite is an understanding of the electrolyte under various conditions. Here we investigate the dependence of the ionic conductivity on lattice strain. Due to different thermal expansion coefficients of the materials in use, the electrolyte already experiences strain. Engineering the strain in such a way that it has a positive effect on the ionic conductivity of the electrolyte will lead to an additional enhancement of the fuel cell efficiency.

Previous investigations ${ }^{2-11}$ on the strain dependence already pointed out the increase in conductivity under expansion of the crystal lattice and a decrease under compression. Four groups ${ }^{2-4,10,11}$ proposed sandwich-type epitaxially grown structures consisting of YSZ and an additional oxide with a larger lattice constant (e.g., $\mathrm{Y}_{2} \mathrm{O}_{3}$ ), one group investigated a single layer YSZ of different thickness on a $\mathrm{MgO}$ substrate ${ }^{9}$ and two other groups applied pressure directly to the elec- trolyte material..$^{5,8}$ In 1998 Suzuki et al. ${ }^{2}$ investigated the ionic motion of oxygen in $\mathrm{YSZ} / \mathrm{CeO}_{2}$ sandwich structures by molecular dynamics simulations. They found an increase of the diffusion coefficient in YSZ by a factor of 1.7 in comparison to bulk conductivity due to the expansive strain the $\mathrm{CeO}_{2}$ layers induce in the electrolyte. In 2005 Kosacki et al. ${ }^{9}$ showed in their experiment that the conductivity of an epitaxial layer YSZ on a $\mathrm{MgO}$ substrate is increased by up to an order of magnitude in comparison to the bulk material, while the activation barrier decreases by a factor of 2 . Three years later Korte et al. $^{3}$ presented an experiment in which they measured the ionic conductivity in $\mathrm{YSZ} / \mathrm{Y}_{2} \mathrm{O}_{3}$ sandwich structures. For increasing numbers of sandwich repetitions (i.e., increasing number of YSZ layers of decreasing thickness) they found an enhancement of the ionic conductivity perpendicular to the layers. From a layer thickness of $200 \mathrm{~nm}$ down to $24 \mathrm{~nm}$ the conductivity increased by a factor of about 1.3 showing a decrease in activation energy of about $0.14 \mathrm{eV}$. In 2009 they reported experiments with three different YSZ/oxide structures for both compressive and expansive strain showing an exponential dependence of the conductivity on the lattice mismatch. ${ }^{4}$ Pennycook et al. ${ }^{10}$ investigated the structure and the ionic conductivity in a zirconia $\left(\mathrm{ZrO}_{2}\right)$ slab sandwiched between strontium titanate by means of molecular dynamics simulations. They found under an expansive strain of $7 \%$ that a new oxygen sublattice structure occurs and estimated the conductivity increase to be six orders of magnitude. Araki et al. ${ }^{5}$ presented an experiment in which they directly exposed the electrolyte material to external expansive pressure. They also observed an increase in ionic conductivity but did not find a change in the activation energy, while Sate et al. ${ }^{8}$ observed in a similar experiment an increase in activation energy under compressive strain. In 2010 Kushima and Yildiz published an extensive investigation ${ }^{6}$ about the strain dependence of 
the migration barrier in YSZ for five different migration configurations based on Density Functional Theory (DFT) computations. They showed for one migration process without any impurities in the direct vicinity that the migration barrier decreases with expansive strain even toward a barrier of height zero. However, for the other more complicated models they could not find a clear behavior, only a rough decreasing trend in most models. A review article ${ }^{12}$ from 2010 covers most of these approaches in a more extensive way.

Here we will investigate the strain dependence of the ionic migration in zirconia based on DFT calculations and provide a conclusive analytic discussion of the phenomenon. We will focus on one basic migration configuration without any correlation effects over a wide range of strain to understand the physics behind the strain dependence. We assess the performance of the electrolyte only from the migration barrier at a given strain. Since the ionic conductivity depends exponentially on the migration barrier $\left[\propto \exp \left(-\Delta E / k_{B} T\right)\right]$, it is the most crucial material quantity. Other material parameters, like the Vineyard coefficient ${ }^{13}$ describing the local vibrational properties of the lattice or the jump distance, affect the conductivity only linearly and will have a minor influence. An exponential dependence of the ionic conductivity with respect to external strain has been reported ${ }^{3,4,6,8-10}$ supporting this kind of procedure.

To understand the DFT results, we have set up a simple atomic model consisting of five ions with Lennard-Jones (LJ) interactions between them. The strain dependence is accounted for by setting the zirconium ion distance corresponding to the one obtained in DFT for each strain value.

In the next section we will describe first-principle computations to calculate the strain dependence. In Sec. III we present the analytic model, which will give insight into the DFT results. A brief discussion of the findings concludes the paper (Sec. IV).

\section{AB INITIO APPROACH}

\section{A. DFT calculations}

Zirconia has three different phases occurring under normal conditions. At low temperatures $(0-1173 \mathrm{~K})$ it is in the monoclinic phase, at intermediate temperatures (1173-2370 K) in the tetragonal phase, and between $2370 \mathrm{~K}$ and the melting point at $2680 \mathrm{~K}$ in the cubic fluorite phase. ${ }^{14}$ The highest ionic conductivity is found in the cubic phase. ${ }^{14}$ Zirconia has to be doped by, for example, yttrium or scandium to stabilize the cubic phase down to room temperature.

To determine the strain dependency of the migration barrier $\Delta E$ for the oxygen ion jumps in zirconia we employ DFT calculations as implemented in the Vienna $a b$ initio package (VASP) using a plane wave basis set. The projector augmented wave (PAW) method by Blöchel has been used, while the exchange and correlation effects were described by the generalized gradient approximation (GGA) using the Perdew-Burke-Ernzerhof (PBE) function. A plane wave basis set up to an energy cutoff of $600 \mathrm{eV}$ has been used, as well as a $3 \times 3 \times 3$ Monkhorst-Pack mesh for the $k$-point sampling. For both, the plane wave basis set and the $k$-point sampling the energy convergence has been tested to guarantee accurate results. Our computational cell is built up by $3 \times 3 \times 3$ unit cells (we also confirmed our results in a $4 \times 4 \times 4$ supercell with a total of 191 ions). The unit cell of the fluorite structure consists of one zirconium and two oxygen ions, leading to a total of 27 zirconium ions, 53 oxygen ions, and one oxygen vacancy. The oxygen atoms have been described by six $\left(2 s^{2} 2 p^{4}\right)$ valence electrons and the zirconium atoms by four $\left(4 d^{3} 5 s^{1}\right)$. We also tested our results using another pseudopotential with 12 valence electrons $\left(4 s^{2} 4 p^{6} 4 d^{3} 5 s^{1}\right)$ for the zirconium atoms, but did not observe a qualitative difference.

To avoid any correlation effects between the vacancy and other defects we investigate a migration process in pure zirconia with only one vacancy in our numerical cell. Since we neglect the effect of the yttrium doping or vacancy-vacancy interactions, it is possible to investigate the pure strain effect on the height of the migration barrier. In YSZ the vacancies are most likely positively charged ${ }^{15,16}$ due to the smaller electron number of yttrium in comparison to zirconium. To obtain these types of vacancies we reduce the total number of electrons in the system. In VASP excess charges are compensated by a homogeneous background to ensure a neutral computational cell.

In the cubic phase of zirconia there are three direct oxygen ion migration pathways possible as shown in Fig. 1. Typically only the first Nearest Neighbor (1NN) jump from one tetrahedron to a neighboring one is considered. This is based on the assumption that the $2 \mathrm{NN}$ and the $3 \mathrm{NN}$ pathways could be neglected. We investigated this by calculating the Minimum Energy Pathway (MEP) between those configurations. The results, as shown in Fig. 2 (as well as the strain dependence of these jump processes discussed in the next section), justify this assumption: The migration barriers (about $3 \mathrm{eV}$ for $2 \mathrm{NN}$ and $4 \mathrm{eV}$ for $3 \mathrm{NN}$, respectively) are much higher than that of the $1 \mathrm{NN}$ jump and can be neglected due to the exponential dependence of the conductivity on the height of the migration barrier.

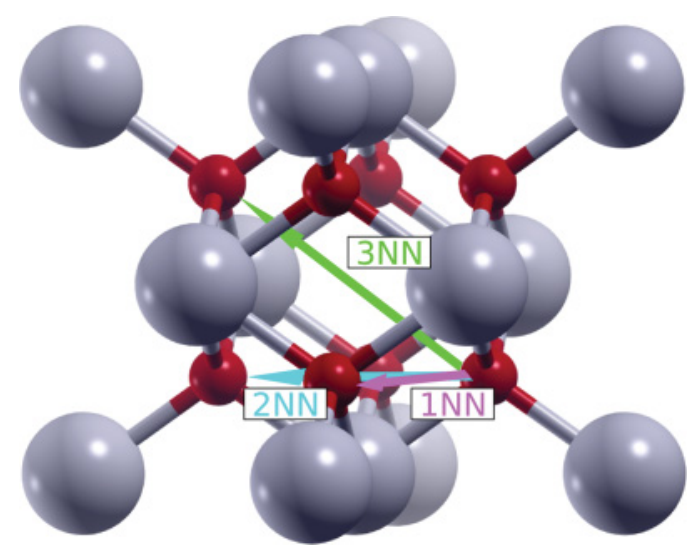

FIG. 1. (Color online) The cubic fluorite phase of zirconia. The pathways for oxygen ion migration are shown as (colored) arrows. The three arrows indicate the possible jump processes to the nearest [1NN (purple)] location and to the next nearest ones [2NN (teal)], [3NN (green)]. A vacancy has to be present at the final position for any of these jumps to take place. (The atomic viewer XCrysDen has been used to create part of this picture. ${ }^{17}$ ) 


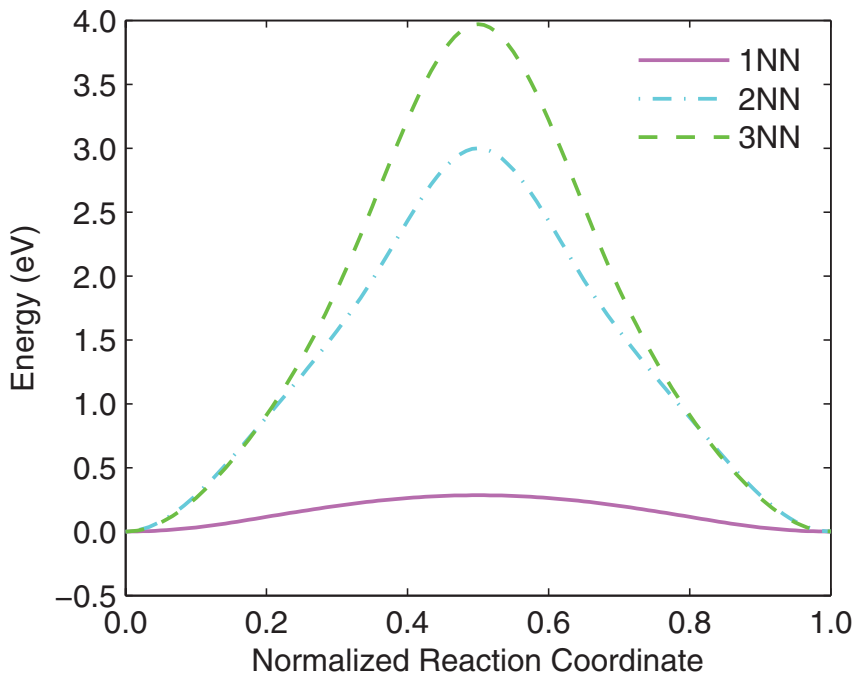

FIG. 2. (Color online) The energies along the three different oxygen ion jump pathways in zirconia calculated in a lattice without external strain. The $2 \mathrm{NN}$ and $3 \mathrm{NN}$ pathways have a considerably larger migration barrier and can thus be neglected.

The strain is introduced by calculating the migration barrier along the $1 \mathrm{NN}$ pathway for different lattice constants. We applied strain isotropically to the system, keeping the cell shape cubic. The factor $\sigma$ scaling the lattice constant in the DFT calculation defines the strain in the system, where $\sigma=1$ denotes the strain-free case. The migration barrier is calculated by using the nudged elastic band (NEB) method, ${ }^{18}$ employing an improved tangent finding method. ${ }^{19}$ In the NEB method first the initial and the final state are relaxed to make sure that the bottom of the potential well is reached. Then these two states are connected by a band of several system images (in our case typically 3 were enough) between which an artificial spring force acts. The initial band is obtained by linearly interpolating all atomic positions between the initial and final state. Then each image is relaxed under the constraint such that they can only move perpendicular to the band and are kept at constant distance along the band by the artificial spring forces. As expected, the maximum energy is reached when the oxygen is located at the brink of two tetrahedrons being exactly between two zirconium ions. As will be described below, only for very large strains this is not the case any more.

\section{B. DFT results}

We find the lattice constant of pure zirconia to be $5.106 \AA$, which is very close to the experimental value ${ }^{20}$ of $5.090 \AA$. We also made test calculations to see the effect of an on-site Coulomb correction on the equilibrium lattice constant. With $U=6 \mathrm{eV}$ and $J=1 \mathrm{eV}$ the lattice constant increased to $5.3 \AA$, which deviates much stronger from the experimental value than our GGA-PBE results. This encouraged us to apply the GGA-PBE formalism, which has the advantage of avoiding further parameters like $U$ and $J$.

Starting from this lattice constant we determine the migration barrier for compression and for expansion. For each strain value we first relax the initial and final position until the maximum force is smaller than $1 \mathrm{meV} / \AA$ and subsequently

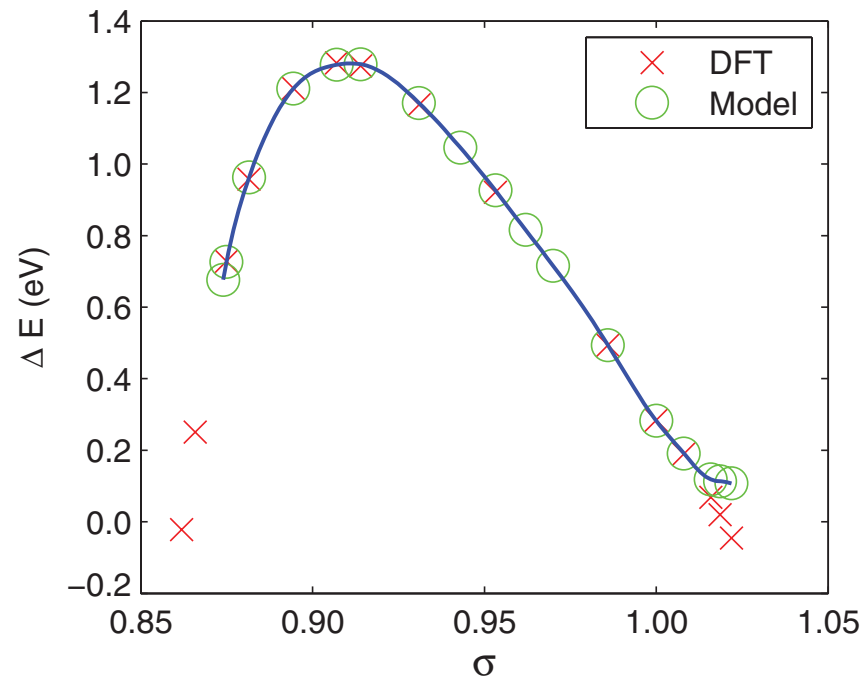

FIG. 3. (Color online) Height of the migration barrier as a function of the strain $\sigma$. The (blue) solid line shows a spline interpolation of the model data. The model agrees over a wide strain range with the DFT results. For $\sigma<0.87$ the model is not valid any more and deviates for $\sigma>1.008$ (see text for discussion).

calculate the height of the migration barrier with the NEB method.

As a result of our strain dependent DFT calculations (cf. Fig. 3) we find that the migration barrier decreases with expansive strain in the neighborhood of the strain-free state, as expected from experiments. However, under compression the height of the migration barrier is not a monotonically increasing function of the strain, but it rather has a maximum at a specific compression value. Under higher compressions the migration barrier decreases again until it vanishes. While it is intuitively clear that the migration barrier decreases under expansion, it was not expected to find a decrease at high compression as well.

We further investigated how the vicinity of a jumping oxygen ion affects the height of the migration barrier. Since the zirconium ions between which the oxygen has to pass move most to let the oxygen pass, we first look into their effect. To find out how costly it is to displace these two ions to the distance necessary for the oxygen ion to hop, we fix these to the barrier-position distance and relax the rest of the lattice. We find that the energy changed by only $11 \mathrm{meV}$ at $\sigma=1$, by $0.4 \mathrm{eV}$ at $\sigma=0.914$, which correspond to the maximal height of the migration barrier, and $0.24 \mathrm{eV}$ at $\sigma=0.866$. This indicates that the local potential acting on the zirconium ions is rather flat, thus the displacement along the corresponding direction is easy. We investigated the effect of further ion sets by constrained NEB, that is, by a NEB calculation with a subset of ions fixed. Two findings can be deduced from these calculations: First, the migration barrier is a very sensitive quantity, which changes strongly when fixing presumably unimportant or distant ions; typically $0.1 \mathrm{eV}$ per fixed ion at $\sigma=0.9$ and $0.4 \mathrm{eV}$ per fixed ion at $\sigma=0.86$. Considering that the migration barrier results from a small difference of large energies (the total energy of the supercell changes by $200 \mathrm{eV}$ in the overall strain range, while the migration barrier changes maximal only by about $1.3 \mathrm{eV}$ ) 
this sensitivity is not surprising. It shows that the jump process is a complex one being affected by not only near but also more distant ions. Such a sensitivity has been reported before. ${ }^{21,22}$ Secondly, it turns out that the migration barrier maximum does not vanish if ions are prevented from relaxing, but it rather shifts to stronger compression values and at the same time increases the migration barrier height at all strains.

It has to be pointed out that for negative migration barriers the system is not in the ground state at initial and final position, therefore the system would deviate away from this configuration. However, these states are metastable and it is thus possible to determine a "negative migration barrier" in the same manner as for positive ones. Although they do not have a deeper physical meaning, they show a consistent behavior of the $\Delta E(\sigma)$ curve even for negative migration barriers.

The migration barrier becomes negative in the regime of small $\sigma$ values. Additionally, for $\sigma \geqslant 1.01$ the lattice becomes sparsely filled leading to the emergence of new stable configurations, which resemble the tetragonal phase in the oxygen sublattice. It is also possible that these configurations correspond to the new phase in the oxygen sublattice reported in Ref. 10 .

In order to check whether the migration barriers of the $2 \mathrm{NN}$ and $3 \mathrm{NN}$ jumps can become small enough to compete with the $1 \mathrm{NN}$ jump under strong compressive strain, we calculated their strain dependence as well. However, these calculations, although showing the same qualitative behavior as the comprehensively investigated $1 \mathrm{NN}$ jump, result in migration barriers, which are overall at least $1-2 \mathrm{eV}$ higher than the corresponding $1 \mathrm{NN}$ value. The $2 \mathrm{NN}$ barrier has a maximum of $3.75 \mathrm{eV}$ and the $3 \mathrm{NN}$ barrier of $4.35 \mathrm{eV}$. Thus the $2 \mathrm{NN}$ and the $3 \mathrm{NN}$ jump can also be neglected at higher compressions.

The DFT computations definitely give the maximum of the migration barrier as a function of $\sigma$, but to gain further understanding we have set up a model which reproduces this unanticipated behavior and provides insight into the physics behind it. In the next section this model will be described and its results discussed.

\section{ANALYTIC APPROACH}

\section{A. Model description}

Because of the Born Oppenheimer theorem the energy of the DFT functional $E_{\mathrm{DFT}}$ is at the same time the potential energy $V$ of the $N$ ions

$$
V\left(r_{1}, r_{2}, \ldots, r_{3 N}\right)=E_{\mathrm{DFT}}\left(r_{1}, r_{2}, \ldots, r_{3 N}\right) .
$$

This potential is not only complicated but the changes of the ionic positions also become complicated as soon as one ion is moving toward a vacancy. The reason is the long-range interaction between the various ions. As noted above, even when only fixing the positions of ions not in the immediate neighborhood of the moving one, the migration barrier will change considerably-by $40 \%$ or more.

This complicated behavior is contrasted with the conjecture that in a motion to a vacancy just two coordinates seem to be essential, one (indicated by $y$ in Fig. 4) being the coordinate of the oxygen ion in the direction of the vacancy,

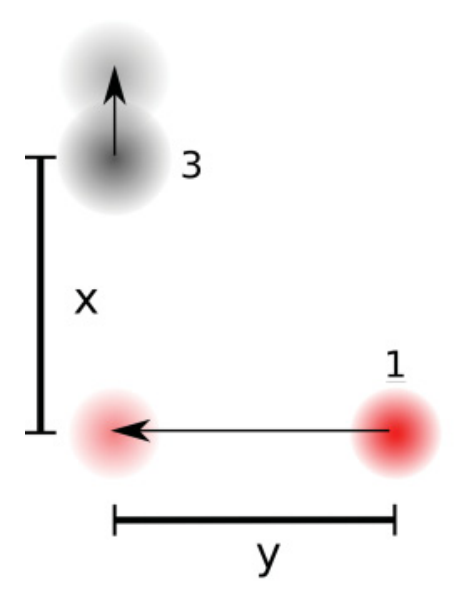

4
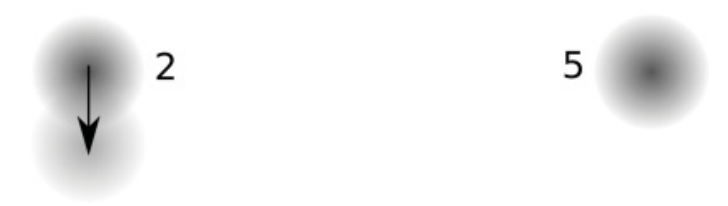

FIG. 4. (Color online) Sketch of the atomic configuration considered in the model (shown two dimensionally). Ion 1 (red) is the oxygen ion, while the ions 2-5 (gray) denote the zirconium ions at the corners of the tetrahedron. The dynamics considered in modeling the jump process is indicated by the arrows. The origin of $x$ and $y$ is the center of mass of the two zirconium ions 2 and 3 .

the other (indicated by $x$ in Fig. 4) being half the distance of the two zirconium ions between which the oxygen ion will pass. However, when all the other coordinates are of marginal importance we can integrate them out in phase space. Because of the exponential dependence this amounts to the replacement

$$
V_{m}(x, y)=\min _{r_{3} \cdots r_{3 N}} V\left(x, y, r_{3} \cdots r_{3 N}\right)
$$

by which we have in fact reduced the problem to the supposed relevant coordinates. It will turn out that in contrast to the long range potential $V$ the potential $V_{m}$ can be represented by a sum of short ranged Lennard-Jones potentials.

Next we can convince ourselves that the coordinates $(x, y)$ are in fact the relevant ones and the potential $V_{m}(x, y)$ the relevant potential. Indeed, if $x$ and $y$ are kept fixed at the saddle point values, all other variables relaxing from the initial positions should also take their saddle point positions. This is in fact the case. Consequently, the NEB method applied to determining the saddle point in this two-dimensional problem will not only lead to the correct initial and saddle point positions but also to the correct energies at these points and consequently to the correct migration barrier as well.

It is therefore tempting to extract the physics out of the potential $V_{m}$ by constructing a simple model with interactions fitting $V_{m}$. This will be described in the following.

When an oxygen ion is moving to a vacancy, a displacement of many ions takes place due to the long-range character of $V$. However, these displacements are expected to lead to a screening with the result that $V_{m}$ is an effective shortrange potential. We will demonstrate this by replacing $V_{m}$ with LJ potentials and taking into account only the direct neighborhood of the moving oxygen ion as shown in Fig. 4. 
This neighborhood consists of four $\mathrm{Zr}^{4+}$ ions forming the corners of a tetrahedron with the $\mathrm{O}^{2-}$ ion initially located in the center. The interaction between zirconium and oxygen is represented by a LJ potential with constant parameters $\epsilon_{\mathrm{Zr}-\mathrm{O}}$ and $r_{\mathrm{Zr}-\mathrm{O}}$. Furthermore, the motion of the $\mathrm{Zr}^{4+}$ ions 4 and 5 in Fig. 4 is neglected being small compared to the motion of the $\mathrm{Zr}^{4+}$ ions 2 and 3. Their motion is being exposed to other ions close by, which we take care of with the following assumptions: These $\mathrm{Zr}^{4+}$ ions can move in the $x$ direction only and their LJ interaction depends on $\sigma$. In DFT we introduced $\sigma$ as the scaling factor for the lattice constant, therefore scaling the side-length of the tetrahedron in the same linear way is a natural choice to simulate compression and expansion in the model. We choose this length to be the in $\sigma$ linearized DFT distances of the $\mathrm{Zr}^{4+}$ ions.

Now we can construct the model potential $V_{M}(x, y)$ :

$$
\begin{aligned}
V_{M}(x, y)= & 2 \epsilon_{\mathrm{Zr}-\mathrm{O}}\left[\left(\frac{r_{\mathrm{Zr}-\mathrm{O}}}{\sqrt{x^{2}+y^{2}}}\right)^{12}-2\left(\frac{r_{\mathrm{Zr}-\mathrm{O}}}{\sqrt{x^{2}+y^{2}}}\right)^{6}\right]+2 \epsilon_{\mathrm{Zr}-\mathrm{O}}\left[\left(\frac{r_{\mathrm{Zr}-\mathrm{O}}}{\sqrt{x_{i}^{2}+\left(2 y_{i}-y\right)^{2}}}\right)^{12}-2\left(\frac{r_{\mathrm{Zr}-\mathrm{O}}}{\sqrt{x_{i}^{2}+\left(2 y_{i}-y\right)^{2}}}\right)^{6}\right] \\
& +\epsilon_{\mathrm{Zr}-\mathrm{Zr}}(\sigma)\left[\left(\frac{r_{\mathrm{Zr}-\mathrm{Zr}}(\sigma)}{2 x}\right)^{12}-2\left(\frac{r_{\mathrm{Zr}-\mathrm{Zr}}(\sigma)}{2 x}\right)^{6}\right]
\end{aligned}
$$

with the initial coordinates $\left(x_{i}, y_{i}\right)$. Since the zirconium ions 4 and 5 do not move and the tetrahedron is symmetric, it is possible to express their distance to the oxygen ion by $\sqrt{x_{i}^{2}+\left(2 y_{i}-y\right)^{2}}$. The distance $r_{\mathrm{Zr}-\mathrm{O}}$ is the equilibrium position of the $\mathrm{Zr}^{4+}-\mathrm{O}^{2-}$ bond in the crystal. This can be determined from the corresponding distances in strain-free DFT calculations. Employing a radial distribution function we find the equilibrium distance to be $2.198 \AA$, which conforms well to the findings reported by Li et al. ${ }^{23}$ The parameter $\epsilon_{\mathrm{Zr}-\mathrm{O}}$ is fixed to $\epsilon_{\mathrm{Zr}-\mathrm{O}}=0.16 \mathrm{eV}$. The values for $r_{\mathrm{Zr}-\mathrm{Zr}}(\sigma)$ and $\epsilon_{\mathrm{Zr}-\mathrm{Zr}}(\sigma)$ are chosen for each $\sigma$ such that (i) the initial configuration and (ii) the resulting migration barrier fit to those obtained from DFT. $\epsilon_{\mathrm{Zr}-\mathrm{Zr}}(\sigma), r_{\mathrm{Zr}-\mathrm{Zr}}(\sigma)$, and $\epsilon_{\mathrm{Zr}-\mathrm{O}}$ together reflect the highly complex impact of the surrounding lattice.

The energy of the model at the initial position is given by

$$
E_{i}=V_{M}\left(x_{i}, y_{i}\right)
$$

while the energy at the barrier position can analogously be described by

$$
E_{b}=V_{M}\left(x_{b}, 0\right),
$$

where the equilibrium distance at the barrier position $x_{b}$ is determined by minimizing the energy with fixed $y=0$.

With Eqs. (4) and (5) the migration barrier

$$
\Delta E_{M}(\sigma)=E_{b}(\sigma)-E_{i}(\sigma)
$$

can be determined.

\section{B. Model results}

In Fig. 3 the $\Delta E(\sigma)$ curve resulting from this model is compared to the DFT values. By adapting $r_{\mathrm{Zr}-\mathrm{Zr}}$ and $\epsilon_{\mathrm{Zr}-\mathrm{Zr}}$ for each $\sigma$ value, it is possible to fit the model curve to the DFT results over a wide domain of strain. Below $\sigma \approx 0.87$ the model cannot describe the situation correctly anymore since the repulsion of the other ions in the vicinity cannot be described anymore by adapting the LJ interaction between the $\mathrm{Zr}^{4+}$ ions. In addition, the model deviates from the DFT curve for $\sigma>1.008$, which corresponds to the occurrence of the new stable configurations in DFT (cf. Sec. II B).
In Fig. 5 the decomposed energies of the model are shown as a function of $\sigma$. Several extrema can be observed near $\sigma=0.95$ : The energy of the $\mathrm{Zr}^{4+}-\mathrm{Zr}^{4+}$ interaction have both a minimum at the initial position $E_{i}(\mathrm{Zr}-\mathrm{Zr})$ and at the barrier position $E_{b}(\mathrm{Zr}-\mathrm{Zr})$. A weak minimum can also be found in the initial energies of the $\mathrm{Zr}^{4+}-\mathrm{O}^{2-}$ interactions, $E_{i}\left(\mathrm{Zr}_{2,3}-\mathrm{O}\right)$ and $E_{i}\left(\mathrm{Zr}_{4,5}-\mathrm{O}\right)$.

On the other hand, at the barrier position the energy of the interaction between the oxygen ion and the zirconium ions 2 and $3 E_{b}\left(\mathrm{Zr}_{2,3}-\mathrm{O}\right)$ has a maximum and a distinct decrease for $\sigma<0.93$. The explanation for this unexpected behavior is an increasing distance $x_{b}$ between the zirconium ions $\mathrm{Zr}_{2,3}^{4+}$ and the oxygen ion (cf. Fig. 4). This increase occurs with compression for $\sigma<0.93$ and has been verified in the DFT calculations.

At the barrier position the energy of the interaction between the oxygen ion and the zirconium ions 4 and $5 E_{b}\left(\mathrm{Zr}_{4,5}-\mathrm{O}\right)$

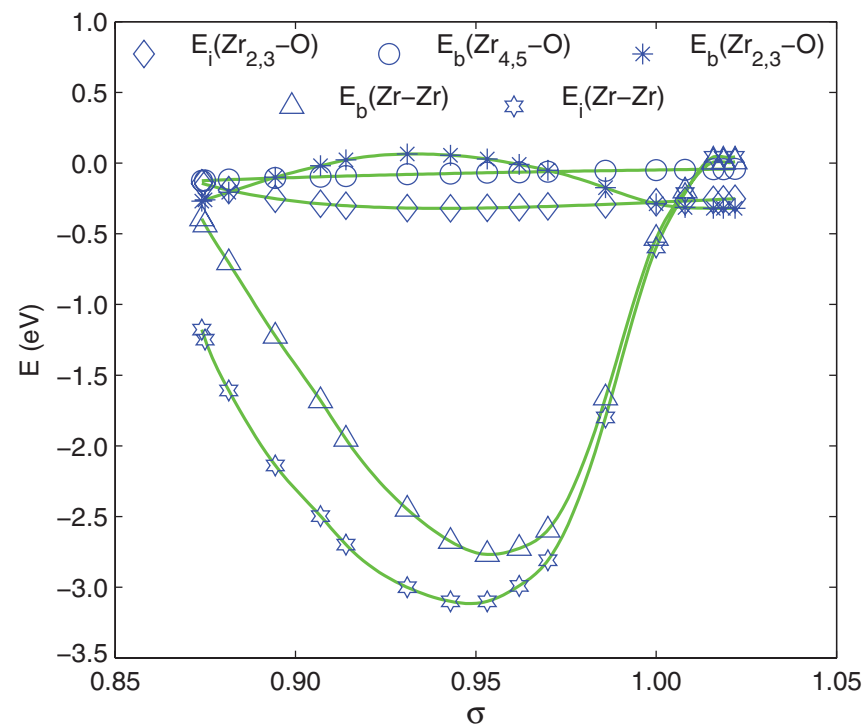

FIG. 5. (Color online) Decomposed initial $E_{i}$ and barrier $E_{b}$ energies in the model as a function of $\sigma$. The energies $E_{i, b}(\mathrm{Zr}-\mathrm{Zr})$ incorporate the $\mathrm{Zr}^{4+}-\mathrm{Zr}^{4+}$ interaction, the energies $E_{i, b}\left(\mathrm{Zr}_{2,3}-\mathrm{O}\right)$ and $E_{i, b}\left(\mathrm{Zr}_{4,5}-\mathrm{O}\right)$ incorporate the $\mathrm{O}^{2-}$ interaction with the corresponding $\mathrm{Zr}^{4+}$ ions. Note: $E_{i}\left(\mathrm{Zr}_{2,3}-\mathrm{O}\right)=E_{i}\left(\mathrm{Zr}_{4,5}-\mathrm{O}\right)$. 
has no extremum at all but decreases continuously with decreasing $\sigma$. This can easily be understood: This bond is always attracting and therefore its energy decreases under compression.

We see now that there are two main reasons for the migration barrier to decrease again at stronger compression although the energy difference of the $\mathrm{Zr}^{4+}-\mathrm{Zr}^{4+}$ bond rises: (a) the initial position becomes more and more unfavorable since all four $\mathrm{Zr}^{4+}-\mathrm{O}^{2-}$ bonds are contracted and thus strongly increasing the energy of the initial oxygen position, whereas the energy at the barrier position does not increase this strongly at large compressions. Furthermore, the potential acting on the zirconium ions in the $x$ direction is not very steep. This result, obtained from the DFT calculations, leads to a slow increase of the difference $E_{b}(\mathrm{Zr}-\mathrm{Zr})-E_{i}(\mathrm{Zr}-\mathrm{Zr})$. (b) The distance between zirconium and oxygen increases again at strong compressions leading to a drop of the oxygen-zirconium repulsion at the barrier position. A further effect, which is present at all compression values, is the decreasing energy $E\left(\mathrm{Zr}_{4,5}-\mathrm{O}\right)$. This effect diminishes the rise of $\Delta E$.

Taken (a) and (b) together our model not only explains why the $1 \mathrm{NN}$ barrier runs through a maximum with decreasing $\sigma$, but the model also gives a qualitative explanation of why the

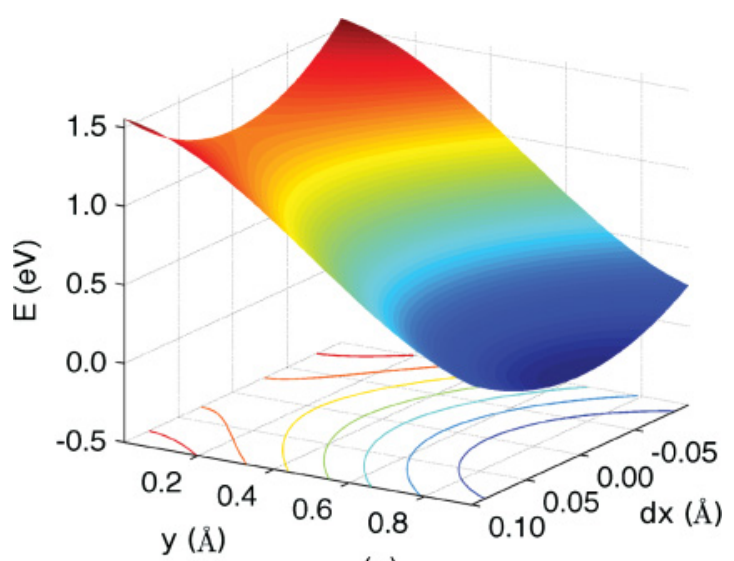

(a)

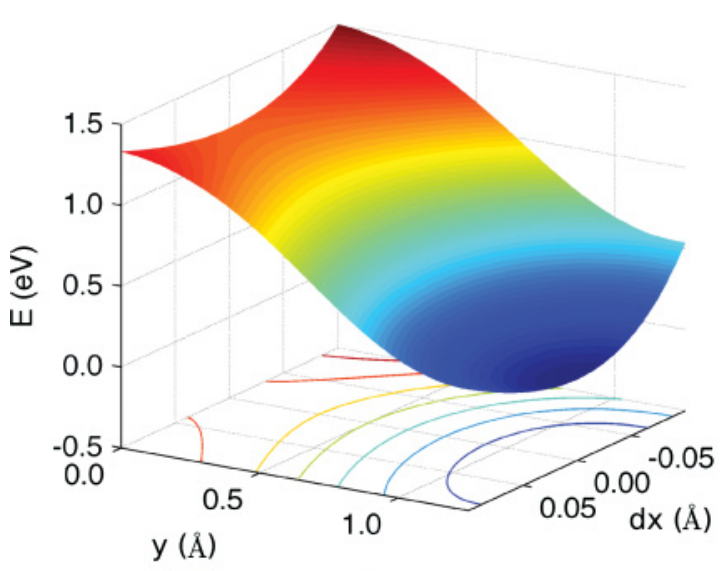

(b)

FIG. 6. (Color online) The effective potential $V_{m}(d x, y)$ at $\sigma=$ 0.93 in harmonic approximation from DFT is shown in (a) and $V_{M}(d x, y)$ from the model in (b). The energy is plotted in dependence of $y$ and $d x$, which is the deviation of the zirconium ions from their equilibrium distance for each $y$. barriers of the $2 \mathrm{NN}$ and $3 \mathrm{NN}$ jumps run through a maximum as well. In fact, these jumps start from the same initial states. As pointed out in (a), the initial position becomes more and more unfavorable and hence maxima of these barriers as a function of $\sigma$ are not surprising. Furthermore, the model also explains why the fixing of ion sets in DFT shifts the migration barrier maximum to stronger compression values. If the initial position is fully relaxed, but one or more ions are fixed when calculating the migration barrier, their missing relaxation gives rise to an additional energy contribution, which is compensated by the increasing initial energy only after further compression.

In Fig. 6 the effective potential $V_{m}(d x, y)$ of the DFT calculation in the harmonic approximation is compared with the potential $V_{M}(d x, y)$ of the model. The energy is plotted in dependence of $d x$ and $y$, where $d x$ is the deviation from the reaction pathway in the $x$ direction. For small deviations $d x$ the two potentials agree satisfactorily. This comparison shows that the model [Eq. (3)] describes the effective potential of the DFT calculations well in the neighborhood of the reaction pathway.

\section{CONCLUSIONS}

We made an investigation on how the migration barrier for oxygen ions in zirconia changes when applying expansive and compressive strain to the lattice. Our DFT calculations show that the migration barrier is not at all a decreasing or increasing function of strain but has a maximum at compressive strain beyond which it continuously descends to a vanishing migration barrier. By setting up a simple model based on short ranged Lennard-Jones interactions we can reproduce and explain this behavior: For expansion and low compression the energy increase at the barrier position dominates and is leading to an increase of $\Delta E$ under compression and a decrease under expansion of the lattice. At stronger compressions two effects arise: first the zirconium ions press intensely on the oxygen ion at the initial position and secondly the zirconium-oxygen repulsion at the barrier position drops due to the fact that their distance increases again under further compression. Together both effects induce a drop of $\Delta E$ when the lattice is strongly compressed.

From the DFT calculations we find that the maximum of the migration barrier occurs at an external pressure of about $130 \mathrm{GPa}$. This pressure would have to be applied to the material to observe the maximum of the migration barrier. Such boundary conditions could be achieved by using a diamond anvil cell (DAC) in which pressures of up to $300 \mathrm{GPa}$ can be produced. ${ }^{24}$ More important, we have found new conditions under which the migration barrier strongly decreases. And it is of importance, we think, to look into this phenomenon in more detail. Work is in progress to investigate how the migration barrier changes if correlations are present in the system (e.g., yttrium at the barrier position).

\section{ACKNOWLEDGMENTS}

The DFT calculations have been performed on the supercomputer JUROPA operated by the Jülich Supercomputing Center at the Forschungszentrum Jülich, Germany. 
*j.hirschfeld@fz-juelich.de

${ }^{1}$ R. Hansch, M. R. R. Chowdhury, and N. H. Menzler, Ceram. Int. 35, 803 (2008).

${ }^{2}$ K. Suzuki, M. Kubo, Y. Oumi, R. Miura, H. Takaba, A. Fahmi, A. Chatterjee, K. Teraishi, and A. Miyamoto, Appl. Phys. Lett. 73, 1502-1504 (1998).

${ }^{3}$ C. Korte, A. Peters, J. Janek, D. Hesse, and N. Zakharov, Phys. Chem. Chem. Phys. 10, 4623 (2008).

${ }^{4}$ N. Schichtel, C. Korte, D. Hesse, and J. Janek, Phys. Chem. Chem. Phys. 11, 3043 (2009).

${ }^{5}$ W. Araki, Y. Imai, and T. Adachi, J. Eur. Ceram. Soc. 29, 2275 (2009).

${ }^{6}$ A. Kushima and B. Yildiz, J. Mater. Chem. 20, 4809 (2010).

${ }^{7}$ M. Sillassen, P. Eklund, N. Pryds, E. Johnson, U. Helmersson, and J. Böttiger, Adv. Funct. Mater. 20, 2071 (2010).

${ }^{8}$ K. Sato, K. Suzuki, R. Narumi, K. Yashiro, T. Hashida, and J. Mizusaki, Jpn. J. Appl. Phys. 50, 055803 (2011).

${ }^{9}$ I. Kosacki, C. M. Rouleau, P. F. Becher, J. Bentley, and D. H. Lowndes, Solid State Ionics 176, 1319 (2005).

${ }^{10}$ T. J. Pennycook, M. J. Beck, K. Varga, M. Varela, S. J. Pennycook, and S. T. Pantelides, Phys. Rev. Lett. 104, 115901 (2010).

${ }^{11}$ N. Schichtel, C. Korte, D. Hesse, N. Zakharov, B. Butz, D. Gerthsen, and J. Janek, Phys. Chem. Chem. Phys. 12, 14596 (2010).
${ }^{12}$ E. Fabbri, D. Pergolesi, and E. Traversa, Sci. Technol. Adv. Mater. 11, 054503 (2010).

${ }^{13}$ G. H. Vineyard, J. Phys. Chem. Solids 3, 121 (1957).

${ }^{14}$ P. Aldebert, J.-P. Traverse, J. Am. Ceram. Soc. 68, 34 (1985).

${ }^{15}$ S. Kasamatsu, T. Tada, and S. Watanabe, Appl. Phys. Expr. 2, 061402 (2009).

${ }^{16}$ G. Stapper, M. Bernasconi, N. Nicoloso, and M. Parrinello, Phys. Rev. B 59, 797 (1999).

${ }^{17}$ A. Kokalj, Comput. Mater. Sci. 28, 155 (2003); code available from [http://www.xcrysden.org/].

${ }^{18}$ D. Sheppard, R. Terrell, and G. Henkelman, J. Chem. Phys. 128, 134106 (2008).

${ }^{19}$ G. Henkelman and H. Jónsson, J. Chem. Phys. 113, 9978 (2000).

${ }^{20}$ E. V. Stefanovich, A. L. Shluger, and C. R. A. Catlow, Phys. Rev. B 49, 11560 (1994).

${ }^{21}$ R. Pornpasertsuk, P. Ramanarayanan, C. B. Musgrave, and F. B. Prinz, J. Appl. Phys. 98, 103513 (2005).

${ }^{22}$ R. Krishnamurthy, Y.-G. Yoon, D. J. Srolovitz, and R. Car, J. Am. Ceram. Soc. 87, 1821 (2004).

${ }^{23}$ J. Li, S. Meng, J. Han, and X. Zhang, Sci. China Ser. E 51, 1858 (2008).

${ }^{24}$ R. J. Hemley and N. W. Ashcroft, Phys. Today 51, 26 (1998). 